with spectroscopic characterization and have not looked comfortable in the pure materials journals such as Journal of Materials Science.

As an added encouragement Journal of Materials Chemistry has no page charges (neither does Chemistry of Materials) and offers 50 free reprints.

The huge development during the 1970 s of new materials for integrated circuit manufacture was mostly concentrated in electrical-engineering departments, while the materials scientists were still worrying about bending paper clips. It will be interesting to see if we miss this next revolution too.

Paul Calvert is in the Department of Materials Science and Engineering, University of Arizona, Tucson, Arizona 85712, USA.

\section{Repeated pleasures}

\section{N. McN Alford}

Journal of Materials Science: Materials In Electronics. Editor Arthur Willoughby. Chapman and Hall. 4/yr. EC 195, US and Canada \$170, elsewhere $£ 103$.

THIS journal has an excellent pedigree. It forms part of the Journal of Materials Science, which has a wide readership, is well presented and is essential reading for anyone with an interest in materials. The new journal is more focused but retains the same format and reproduction quality as its parent. It is published quarterly and is free if you already receive Journal of Materials Science. I hesitate to suggest to anyone that they should pick up yet another journal, but this one filis a specialist niche - that of the use of materials in electronics - and for this reason it is likely to be successful. In fact, this means that its territory is the whole range of materials from metals through ceramics to polymers, and the issues I looked at covered a wide range of their electrical and electronic properties. These included ionic and electrical conductivities, dielectric, thermoelectric, photoconductive and ferroelectric properties, as well as properties of superconducting materials. Preparative techniques and properties of the materials were discussed with special emphasis on the electronics. The editorial in the first issue emphasized the importance of applications-based research. And sure enough, I found much science that will be useful in applications, although only one or two of these were mentioned specifically.

The most serious competition to the new publication will come from Journal of Applied Physics and Applied Physics
Letters, both of which discuss the materials aspects of electronic materials. Some journals, such as Journal of Physics and Condensed Matter and Electronics Letters, generally assume that materials science is a necessary chore but not really worth examining in detail. In a sense this is understandable - these are journals about physics or electronics. But when things begin to go wrong it is usually a materials problem. A shining example of this can be found with hightemperature superconductors. Here the processing of the materials, the choice of substrates, the orientation of the superconductor and the substrates, the introduction of weak links and the elimination of weak links, are all materials problems. To be fair, most 'pure' physicists or chemists are not that pure. They have all had to tackle such problems from time to time, which indeed always require a multidisciplinary approach.

The content range of the new journal is excellent, and to the researcher interested in electronics, the juxtaposition of papers on such a broad spread of materials will be fascinating. The only reservation $I$ had was that in some cases I had read very similar material by the

\section{Mature matters}

\section{John Nicholson}

Journal of Materials Science: Materials in Medicine. Editors W. Bonfield and D.F. Williams. Chapman and Hall. 6/yr. EC $£ 135$, US and Canada $\$ 240$, elsewhere 1145 (institutional); members' rates available on application to publisher.

IN recent years the study of materials for use in medicine and dentistry has developed dramatically. Just over 30 years ago, J. Charnley, the orthopaedic surgeon, devised substantial improvements in hip-replacement surgery yet used denture-grade poly(methylmethacrylate) to secure his new prostheses in place. There was little understanding of the mechanical or biological properties needed. All that has changed now. The interdisciplinary science of medical materials has come of age.

To mark this maturity, the new publication Materials in Medicine has been launched, the first edition appearing in June 1990. It is published in association with the well-established Journal of Materials Science and aims to cover the science of the metals, ceramics, polymers and composites used in a range of medical areas, including orthopaedics, maxillofacial and cardiovascular surgery and dentistry. Publication started as quarterly, but is now every two months.

The first editions more than fulfil the same authors in other journals. The solution is tighter refereeing.

The one review article I saw was on radiation damage in solar cells by $T$. Markvart. This was very good and one hopes that such quality reviews can be maintained. In one or two other articles I was struck with the thought: why have the authors done this and is it useful? For example, much is known about plasma spraying and there have been several papers on the application of this technique to oxides. The paper by Hibino et al. on plasma spraying of oxide superconductors in the first issue was therefore not particularly novel. So why include it? More importantly, what are the electronic properties of the product?

The addition of yet another journal requires serious soul-searching. In this case those who get to see the new arrival as part of its parent journal and who are interested in electronics will be very pleased. For others, it is well worth reading a few copies and then deciding whether to subscribe.

N. McN Alford is in the Advanced Materials Division of $\mathrm{ICl}, \mathrm{PO}$ Box 11, The Heath, Runcorn, Cheshire WA7 $4 Q E$, UK.

publisher's aims, and cover work ranging from the porosity of zinc polycarboxylate dental cements to surface modification of medical grade PVC, and from preparation of synthetic hydrozyapatite to enzymic hydrolysis of biopolymers. The quality of these papers is high and the authors can have no grumbles about the time between acceptance and publication, which in most cases was under six months. I will watch with interest to see if this speed can be maintained in the future.

So far, so good. But I must sound one note of discord. There are now many journals operating in this field (Clinical Materials, Biomaterials, Journal of Biomaterials Science, all from the United Kingdom; plus the Journal of Biomaterials Science from the United States) together with a number of specialized journals on the narrower subject of dental materials.

Having refereed papers for several of these journals, I find it hard to believe that there are sufficient people doing work in biomaterials of the necessary quality to sustain all these publications. Consequently, some of these titles may well not survive. If that is so, I hope Materials in Medicine is one that does continue. Given the quality of the first few editions, I think the editors, Bill Bonfield and David Williams, deserve to succeed.

John W. Nicholson is at the Laboratory of the Government Chemist, Queens Road, Teddington, Middlesex TW11 OLY, UK. 